\title{
Evaluation of Quench Hardening in Steel from Three-Dimensional Magnetic Flux Vectors
}

\author{
Keita OGURA, Shigeo KOTAKE and Yasuyuki SUZUKI \\ Department of Mechanical Engineering, Mie University, Mie, Japan \\ e-mail: kotake@mach.mie-u.ac.jp
}

\begin{abstract}
Since austenite shows paramagnetic properties and ferrite and martensite do ferromagnetic ones, magnetic evaluation can be a good candidate for detecting phase transition. However, hence coercive force cannot distinguish ferrite from martensite under external magnetic field, magnetic measurement has not been used for quench hardening evaluation. By contrast, ferromagnetic specimens have inverse-magnetostriction properties, which can cause spontaneous magnetization under phase-transition stress during quenching process. Therefore, in this study, we have tried to develop a technique for quench hardening distribution analysis by sensing spontaneous magnetization caused from inverse-magnetostriction. From the results of the experiments, large spontaneous magnetizations were appeared in the specimen, which indicates partial martensite transformation in that area. However, no residual magnetizations were observed in whole martensite or ferrite phase. Especially, high magnetic intensity was obtained in the area of multiple phases with residual austenite and martensite. The residual stress caused from the different molar volume between fcc austenite and bct martensite phase has an influence to the inverse magnetostriction phenomena emerging high spontaneous magnetization after quenching. Key words: martensite, quenching, inverse-magnetostriction, phase transition stress
\end{abstract}

\section{INTRODUCTION}

To obtain quench hardened iron and steel, it is necessary to yield martensite transformation with high quenching speed before achieving ferrite region $[1,2]$. When the quenching condition is insufficient, it causes problems in practical use.

On the other hand, as an evaluation method for quench hardening of iron and steel, several techniques have been proposed, such as electric resistance measurement, induced electromotive force from eddy current and Vickers hardness measurement. Because the perfect inspection or the local area evaluation is difficult in these methods, some problems happen by using them in manufacturing lines in factories. Moreover, since all these methods can merely evaluate at the vicinity of the surface, so they cannot characterize quench hardening in the bulk region.

Since austenite has paramagnetic properties and ferrite and martensite have ferromagnetism, magnetic measurement can be a good candidate for detecting phase transition of martensite. However, hence coercive force cannot distinguish ferrite from martensite under external magnetic field; magnetic evaluation has not been used for quench hardening measurement.

By contrast, ferromagnetic specimens have inverse-magnetostriction properties, which can be appeared under phase-transition stress during quenching process. Therefore, in this study, we have tried to develop the technique for quench hardening distribution analysis by measuring spontaneous magnetization from inverse-magnetostriction [3].

\section{MEASUREMENT PRINCIPLE}

Magnetic energy, $E$, consists of magnetoelastic $\operatorname{energy}\left(E_{\mathrm{e}}\right)$ and magnetic field energy $\left(E_{\mathrm{m}}\right)$, as show in the next equation between the stress and magnetization in ferromagnetic substance. The magnetic field will change its distribution to minimize $E$ under the stress.

$$
E=-\frac{3}{2} \lambda \sigma\left(\cos ^{2} \theta-\frac{1}{3}\right)+\frac{1}{2 \mu} \mathrm{B}^{2}=E_{e}+E_{m}
$$

where $\lambda$ is magnetostriction coefficient. $\lambda$ of the iron is strongly dependent of crystal orientation. In random orientation, $\lambda$ is positive. $\sigma$ is the stress vector, $\theta$ is with the angle between $\sigma$ and the magnetic flux vector. And $\mu$ is magnetic permeability coefficient in the medium.

Hence magnetic flux distribution is determined by minimizing $E$, we can estimate stress distribution by evaluating an emerged magnetic field. When $E$ decreases under the stress,

$\frac{\partial E}{\partial \sigma}=-\frac{3}{4} \lambda\left\{1-\left(1-2 \frac{\partial \theta}{\partial \partial}\right) \sin 2 \theta\right\}<0$

$\theta$ will also change,

$\frac{\partial \theta}{\partial \sigma}<\frac{1}{2}\left(1-\frac{1}{\sin 2 \theta}\right) \leq 0$

\section{EXPERIMENTS}

\subsection{SPECIMENS}

I used high hardenability alloy tool steel SKD11 and medium hardenability alloy tool steel SKS93. Size of the samples is $25 \mathrm{~mm} \times 25 \mathrm{~mm} \times 10 \mathrm{~mm}$. 


\subsection{EXPERIMENTAL PROCEDURE}

To get rid of the influence of initial magnetization, we make the samples homogeneous and demagnetized by annealing. The annealing condition is shown in Table 1 .

Since the center of the sample was heated locally by an infrared furnace, part of the sample was quenched.

Heating time by the infrared furnace was $200 \mathrm{~s}, 400 \mathrm{~s}$, 800 s and 1600 s. Local quenched area of sample by the infrared furnace is shown in Fig.1.

Table 1 Annealing temperature

\begin{tabular}{|l|l|}
\hline SKD11 & furnace cooling from $950 \square$ \\
\hline SKS93 & furnace cooling from $750 \square$ \\
\hline
\end{tabular}

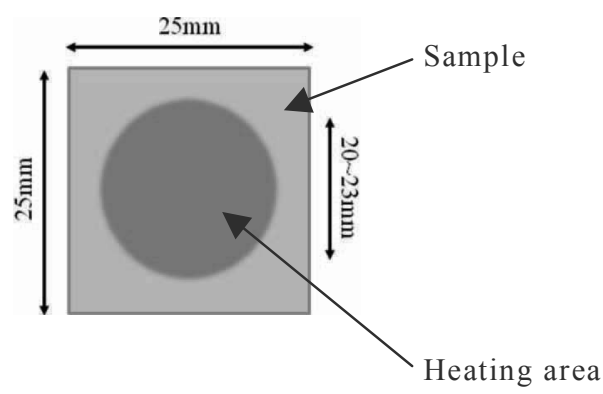

Fig.1 local quenched area of the sample by the infrared furnace

\subsection{MEASUREMENT METHOD}

The diagrammatic view of the leaked magnetization measurement is shown in Fig.2.The apparatus consists with $\mathrm{X}-\mathrm{Y}$ stage and Hall device type gauss meter and magnetic impedance (MI) type gauss meter put on the probe tip for three-axial magnetic field measurement. The sample was placed on the stage inside a permalloy pipe as a magnetic shield. Sample was step scanned in $\mathrm{x}-\mathrm{y}$ direction in every $0.5 \mathrm{~mm}$ by controlling from a PC. Then the distributions of leaked magnetic flux vectors were measured at each position. Distance between magnetic sensor and the sample surface was $1 \mathrm{~mm}$.

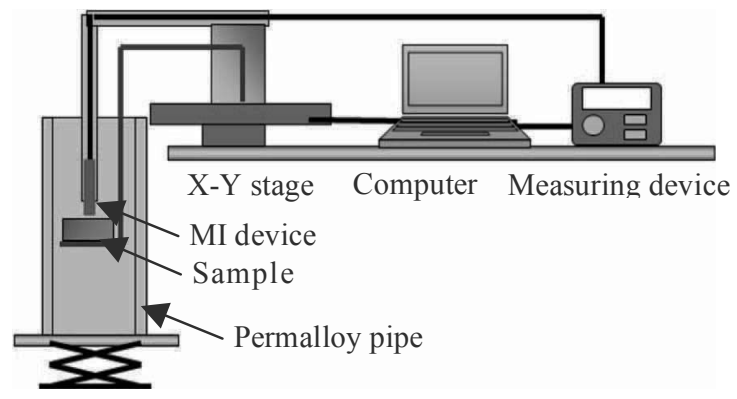

Fig.2 Measurement apparatus of leaked magnetic flux vectors

And we also measure the Vickers hardness to make sure the martensite transformation.

\section{RESULTS AND DISCUSSIONS \\ 4.1 ANNEALING}

Leaked magnetic distribution of the annealed SKD11 is shown in Fig. 3 and that of annealed SKS93 is shown
Fig.4. The shade colors indicate the absolute value of the magnetic flux vector is large. Because the residual magnetic distribution at the annealed SKD11 surface was uniform, and the maximum magnetization was $0.023 \mathrm{mT}$, which is almost equal to the value of terrestrial magnetism $0.024 \mathrm{mT}$, the homogenization processing and the demagnetization processing of the sample was well completed. In the case of annealed SKS93, because the maximum intensity of the magnetization, $3.107 \times 10^{-3} \mathrm{mT}$, was much smaller than the terrestrial magnetism, the homogenization and the demagnetization processing of the sample were completed too.
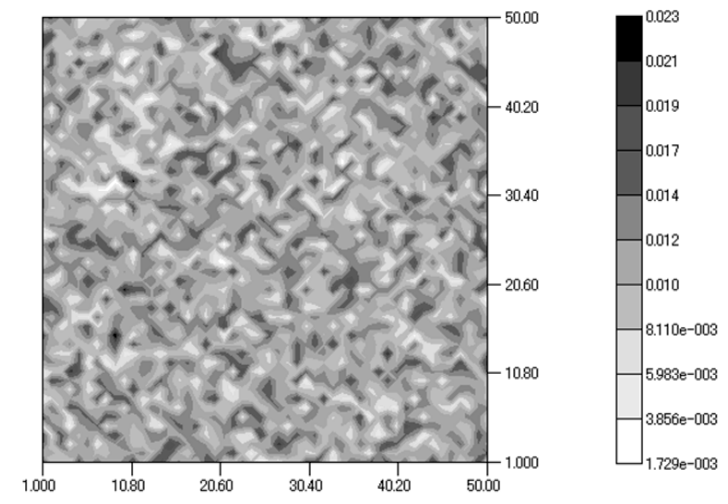

[mT]

Fig.3 Magnetic distribution of the annealed SKD11
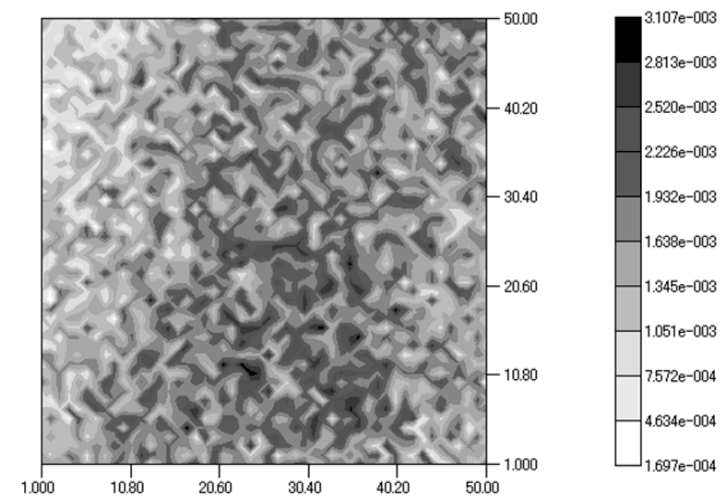

[mT]

Fig.4 Magnetic distribution of the annealed SKS93

\subsection{SKD11 SPECIMENS}

Magnetic distributions of SKD11 after local quenching with each heating time by the infrared furnace are shown in Fig.5, Fig.6, Fig.7, and Fig.8. Since all the maximum values are smaller than the terrestrial magnetism the demagnetization was completed, which indicates heating temperature was above the Curie point of iron, $1043 \mathrm{~K}$, when the samples were heated by the infrared furnace.

In the case of 200 s heating time, the maximum magnetization was $3.590 \times 10^{-3} \mathrm{mT}$ and minimum one was $1.405 \times 10^{-4} \mathrm{mT}$. Right side of the sample with the high residual magnetization shows high Vickers hardness around $919 \mathrm{Hv}$. And, the areas with weak magnetization show low Vickers hardness around $636 \mathrm{Hv}$. In the case of 
400s heating time, same results were obtained. Although certain magnetization was observed, contrast of distribution map is not so clear.

On the contrary, in the case of 800 s as heating time, maximum magnetization was $7.611 \times 10^{-3} \mathrm{mT}$ and minimum one was $1.405 \times 10^{-4} \mathrm{mT}$. Areas of the lower right corner of the sample show strong residual magnetization. Vickers hardness of the same area is around $1067 \mathrm{Hv}$. And the other areas show weak Vickers hardness, around $762 \mathrm{Hv}$. In the case of $1600 \mathrm{~s}$, maximum magnetization was $4.284 \times 10^{-3} \mathrm{mT}$ and Vickers hardness of the same area shows $857 \mathrm{Hv}$.

Moreover, when the heating time becomes longer, the maximum of the residual magnetization becomes higher. From these results, we concluded the strong residual magnetization was generated by the phase-transition stress caused from the martensite and residual austenite mixed phases. And the weak residual magnetic fluxes were observed in ferrite mono phase. This indicates long enough heating time, over $800 \mathrm{~s}$, is necessary for the specimens to reach austenite phase.
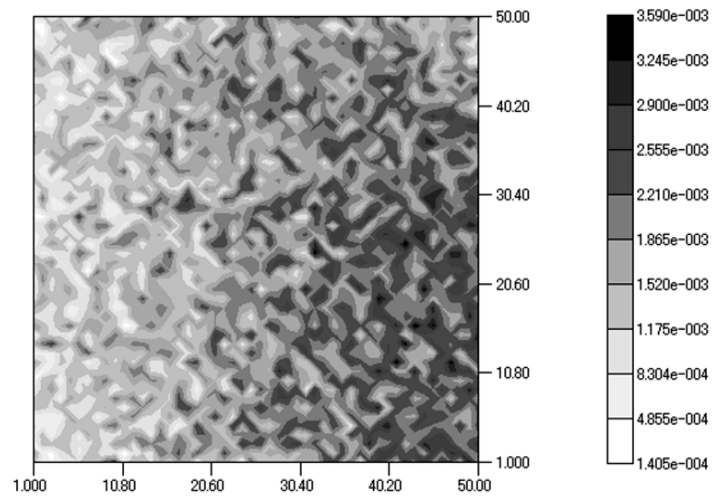

$[\mathrm{mT}]$

Fig.5 Magnetic distribution of 200s locally heated SKD11 by the infrared furnace
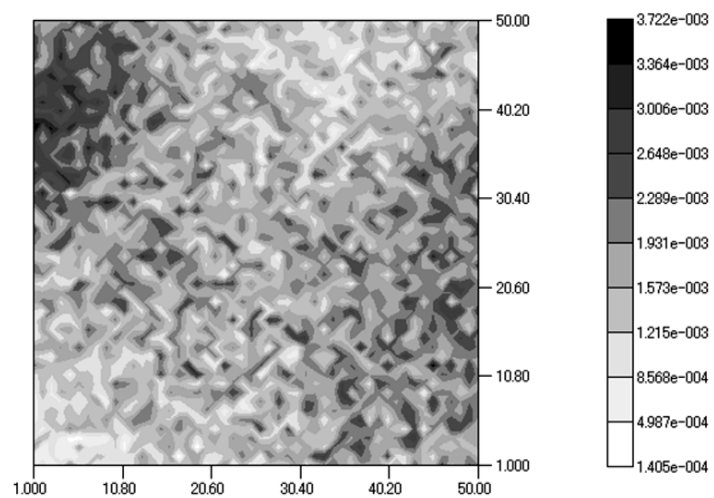

$[\mathrm{mT}]$

Fig.6 Magnetic distribution of 400 s locally heated SKD11 by the infrared furnace
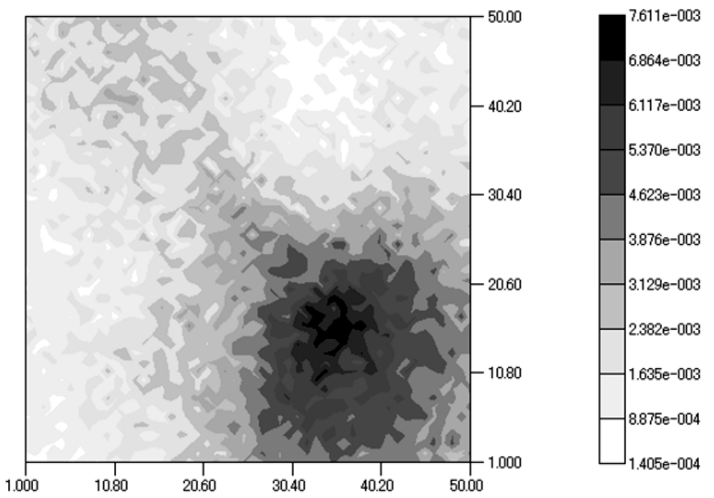

$[\mathrm{mT}]$

Fig.7 Magnetic distribution of 800 s locally heated SKD11 by the infrared furnace
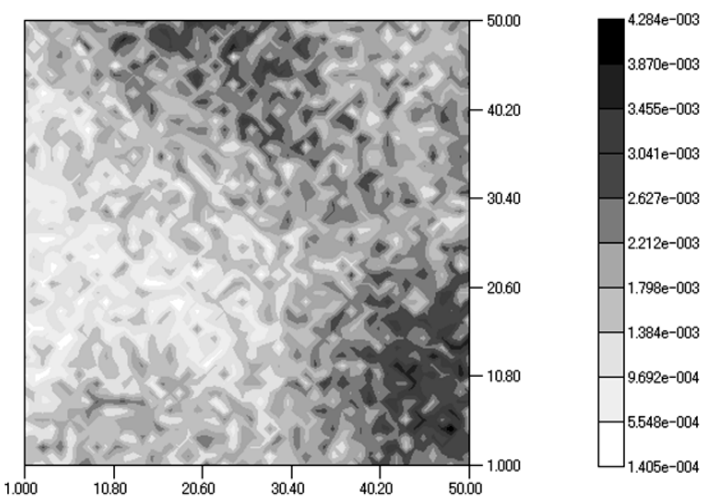

[mT]

Fig. 8 Magnetic distribution of 1600 s locally heated SKD11 by the infrared furnace

\subsection{SKS93 SPECIMENS}

Magnetic distributions of locally heated SKS93 by the infrared furnace are shown in Fig.10, Fig.11, Fig.12, and Fig.13.

In the case of $200 \mathrm{~s}$ heating time, the maximum magnetization was $2.909 \times 10^{-3} \mathrm{mT}$ and minimum one was $1.405 \times 10^{-4} \mathrm{mT}$. All areas of the sample show weak residual magnetizations. Vickers hardness at the all areas of sample is about $416 \mathrm{Hv}$. In the cases of $400 \mathrm{~s}$ and $800 \mathrm{~s}$ heating time, the maximum magnetization was small and Vickers hardness is not large.

On the contrary, in the case of $1600 \mathrm{~s}$, the maximum magnetization was $8.380 \times 10^{-3} \mathrm{mT}$ and the minimum one was $9.007 \times 10^{-4} \mathrm{mT}$. Areas of the lower right corner and the top right corner of the sample show strong residual magnetization. Vickers hardness of the high magnetization areas shows high hardness about $722 \mathrm{Hv}$. And, in the other areas the hardness was weak around $518 \mathrm{Hv}$.Since harden ability of SKS93 was lower than that of SKD11. The strong residual magnetic induction of $400 \mathrm{~s}, 800 \mathrm{~s}$ and $1600 \mathrm{~s}$ were spontaneously generated in SKD11 under the high phase transition stress with mixed martensite and residual austenite. 

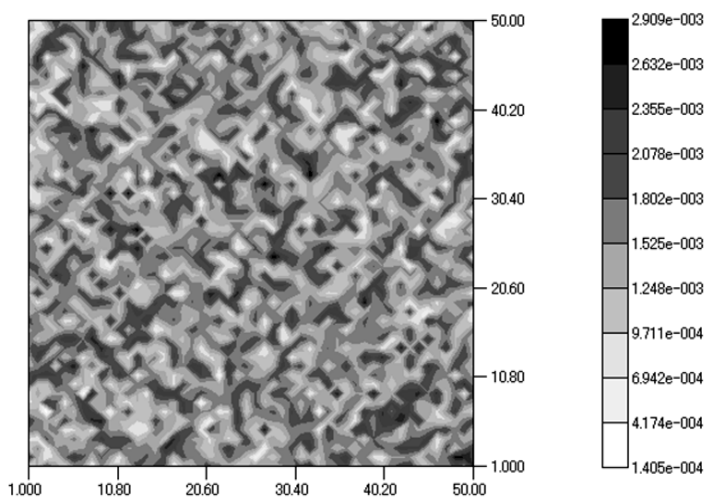

[mT]

Fig.9 Magnetic distribution of 200s locally heated SKS93 by the infrared furnace
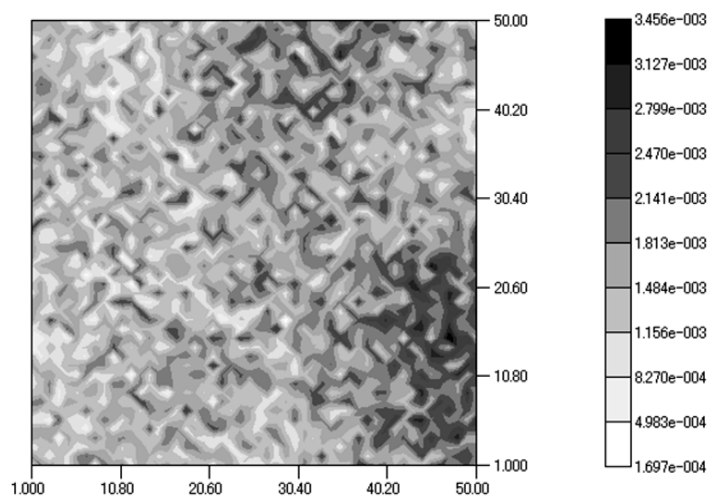

[mT]

Fig.10 Magnetic distribution of 400s locally heated SKS93 by the infrared furnace
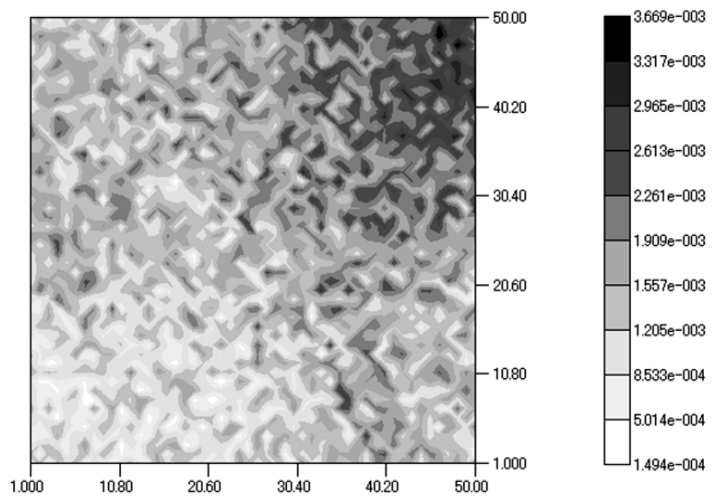

$[\mathrm{mT}]$

Fig.11 Magnetic distribution of 800s locally heated SKS93 by the infrared furnace
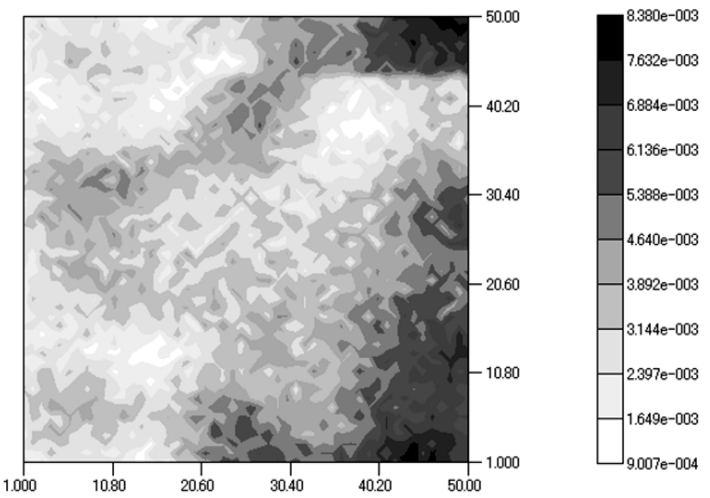

$[\mathrm{mT}]$

Fig.12 Magnetic distribution of 1600 s locally heated SKS93 by the infrared furnace

\section{CONCLUSION}

From the above experimental results, we concluded that quench hardening distribution of steel alloy can be evaluated from three-dimensional magnetic flux vectors, since high Vickers hardness area corresponds strong residual magnetic flux vectors, and low HV corresponds the weak residual magnetization. Moreover, we conclude inverse-magnetostriction occurred under high phase-transition stress, since the distribution of spontaneous magnetization corresponds mixed structure of martensite and residual austenite created by quenching.

\section{REFERENCE}

[1] T. Inoue, K. Haraguchi, S. Kimura, J. Soc. Mater. Sci. Jpn., 25, 521 (1975).

[2] Z. Nishiyama, "The aspect metamorphosis of the iron and steel", AGUNE Technology Center, Tokyo (1975).

[3] H. Hase, S. Kotake, M. Ohota, H. Kawakami, Y. Suzuki, Key Eng. Mater. 340-341, 549 (2007).

(Received December 10, 2008 ; Accepted February 2, 2009) 